\title{
PRIMITIVE ZEROS OF QUADRATIC FORMS MOD $P^{2}$
}

\author{
ALI H. HAKAMI
}

\begin{abstract}
Let $Q(\mathbf{x})=Q\left(x_{1}, x_{2}, \ldots, x_{n}\right)$ be a quadratic form with integer coefficients, $p$ be an odd prime and $\|\mathbf{x}\|=\max _{i}\left|x_{i}\right|$. A solution of the congruence $Q(\mathbf{x}) \equiv \mathbf{0}\left(\bmod p^{2}\right)$ is said to be a primitive solution if $p \nmid x_{i}$ for some $i$. In this paper, we seek to obtain primitive solutions of this congruence in small rectangular boxes of the type $\mathscr{B}=\left\{\mathbf{x} \in \mathbb{Z}^{n}:\left|x_{i}\right| \leq\right.$ $\left.M_{i}, 1 \leqslant i \leqslant n\right\}$ where for $1 \leq i \leq l$ we have $M_{i} \leq p$, while for $i>l$ we have $M_{i}>p$. In particular, we show that if $n \geq 4, n$ even, $l \leq \frac{n}{2}-2$, and $Q$ is nonsingular $(\bmod p)$, then there exists a primitive solution with $x_{i}=0,1 \leq i \leq l$, and $\left|x_{i}\right| \leq 2^{\frac{4 n+3}{n-l}} p^{\frac{n}{n-l}}+1$, for $l<i \leq n$.
\end{abstract}

\section{Introduction}

Let $Q(\mathbf{x})=Q\left(x_{1}, x_{2}, \ldots, x_{n}\right)$ be a quadratic form with integer coefficients and $p$ be an odd prime. Set $\|\mathbf{x}\|=\max \left|x_{i}\right|$. When $n$ is even we let $\Delta_{p}(Q)=\left((-1)^{n / 2} \operatorname{det} A_{Q} / p\right)$ if $p \nmid \operatorname{det} A_{Q}$ and $\Delta_{p}(Q)=0$ if $p \mid \operatorname{det} A_{Q}$, where $(\cdot / p)$ denotes the Legendre-Jacobi symbol and $A_{Q}$ is the $n \times n$ defining matrix for $Q(\mathbf{x}) . Q(\mathbf{x})$ is called nonsingular $(\bmod p)$ if $p \nmid \operatorname{det} A_{Q}$.

Consider the congruence

$$
Q(\mathbf{x})=Q\left(x_{1}, x_{2}, \ldots, x_{n}\right) \equiv 0 \quad(\bmod m)
$$

where $m$ is a positive integer. There has been much interest in obtaining a small nonzero solution of the congruence (1). The problem of finding a small solution of (1) means finding a nonzero integral solution $\mathbf{x}$ such that $\|\mathbf{x}\| \leq m^{\delta}$ for some positive constant $\delta<1$. The constant $\delta$ may depend on $n$, but not on $m$.

In this paper we are seeking to find primitive solutions of (1) in a more general box centered at the origin, in the case where $m=p^{2}$. A primitive solution is one with $\operatorname{gcd}\left(x_{1}, \ldots, x_{n}, m\right)=$ 1. A primitive solution is sought to rule out trivial solutions of (1) of the type $p \mathbf{y}$ where $\mathbf{y}$ satisfies $Q(\mathbf{y}) \equiv 0(\bmod p)$. First, we give some background on what is already known for the case of small solutions. 
For the quadratic form $Q(x)=x_{1}^{2}+\cdots+x_{n}^{2}$, it is clear that any nonzero solution $\mathbf{x}$ of (1) must satisfy, $\max \left|x_{i}\right| \geq \frac{1}{\sqrt{n}} m^{1 / 2}$. Thus $\delta=1 / 2$ is the best possible exponent for a small solution in general.

Schinzel, Schlickewei and Schmidt [17] proved that (1) has a nonzero solution with $\|\mathbf{x}\|<$ $m^{(1 / 2)+1 / 2(n-1)}$ for $n \geq 2$, even, and $\|\mathbf{x}\|<m^{(1 / 2)+(1 / 2 n)}$ for $n \geq 2$, odd. Thus for any $\varepsilon>0$ we get a nonzero solution of (1) with $\|\mathbf{x}\|<m^{(1 / 2)+\varepsilon}$ provided $n$ is sufficiently large. We note that the solution obtained by their method is not necessarily a primitive solution. Indeed, when $m=p^{2}$ they would simply use a trivial solution such as $(p, 0, \ldots, 0)$.

Dealing with $m=p, p$ an odd prime, Heath-Brown [15] obtained a nonzero solution of (1) with $\|\mathbf{x}\| \ll p^{1 / 2} \log p$ for $n \geq 4$. His result was an improvement on the result of [17] in this case. Wang Yuan [18], [19] and [20] generalized Heath-Brown's work to all finite fields. Cochrane, in a sequence of papers [1], [2] and [3] improved this to $\|\mathbf{x}\|<\max \left\{2^{19} p^{1 / 2}, 2^{22} 10^{6}\right\}$. The best constant available is due to the author [7, Theorem 1.3] and [11, Theorem 1] who obtained $\|\mathbf{x}\|<\min \left\{p^{2 / 3}, 2^{19} p^{1 / 2}\right\}$.

Using the method of exponential sums the author [8, Theorem 1] generalized Cochrane's method to find a primitive solution of (1) with $\|\mathbf{x}\| \ll p$ for $n \geqslant 4$ when $m=p^{2}$ and $Q(\mathbf{y})$ is nonsingular $(\bmod p$ ). The optimal bound, $\|x\| \leqslant p$ for $n \geqslant 1$, was obtained by Cochrane and Hakami using a geometric method [6, Theorem 1].

For $m=p^{3}$, the author [9, Theorem 1]. obtained the existence of a primitive solution of any nonsingular form with $\|\mathbf{x}\| \ll p^{(3 / 2)+(3 / n)}$, provided $n \geqslant 6$.

For a general prime power $m=p^{k}$ and nonsingular form $\left(\bmod p^{k}\right)$ in $n \geqslant 4$ variables $(n$ even) a primitive solution of size $\|\mathbf{x}\| \ll m^{(1 / 2)+(1 / n)}$ is obtained by the author [10, Theorem 1].

For $m=p q$ a product of two distinct primes, the optimal bound, $\|\mathbf{x}\| \ll m^{1 / 2}$ for $n>4$ was obtained by Cochrane [4] and [5], building upon the work of Heath-Brown [14]. But no attempt was made to obtain a primitive solution in this work.

As we mentioned our interest in this paper is the case $m=p^{2}$ with $p$ a prime. We wish to obtain the existence of primitive solutions of the congruence

$$
Q(\mathbf{x})=Q\left(x_{1}, x_{2}, \ldots, x_{n}\right) \equiv 0 \quad\left(\bmod p^{2}\right),
$$

in a box of points of the type

$$
\mathscr{B}=\left\{\mathbf{x} \in \mathbb{Z}^{n}:\left|x_{i}\right| \leq M_{i}, \quad 1 \leq i \leq n\right\},
$$

centered about the origin, where $M_{i} \in \mathbb{Z}$, and $0 \leqslant M_{i} \leqslant \frac{p^{2}-1}{2}$ for $1 \leqslant i \leqslant n$. We shall assume that exactly $l$ of the edges are of length at most $p$, while the remaining edges all have lengths strictly greater than $p$, say

$$
2 M_{i}+1 \leq p, \quad 1 \leq i \leq l, \quad 2 M_{i}+1>p, \quad l+1 \leq i \leq n .
$$


We also restrict our attention to the case where $n$ is even and $Q$ is nonsingular $(\bmod p)$, so that $\Delta_{p}(Q)$ is as defined in the opening paragraph.

For the case $\Delta_{p}(Q)=1$, we establish in Corollary 1 that if $n$ is even, $n \geq 4$,

$$
|\mathscr{B}| \geqslant 2^{4 n+2} p^{n}, \quad \text { and } \quad \prod_{i=1}^{l} \frac{p}{2 M_{i}+1} \leqslant 2^{-4 n-2} p^{(n / 2)-1},
$$

(where the product is set equal to 1 if $l=0$ ), then there exists a primitive solution of (2) in the box $\mathscr{B}+\mathscr{B}$, that is, a primitive solution with $\left|x_{i}\right| \leq 2 M_{i}, 1 \leq i \leq n$. A similar result (where $4 n+2$ is replaced by $4 n+3$ ) is established in Corollary 2 for the case $\Delta_{p}(Q)=-1$. In the case where the first $l$ edges are all of length zero, we deduce the following theorem.

Theorem 1. Let $p$ be an odd prime, $Q$ be a quadratic form over $\mathbb{Z}$ in $n \geq 4$ variables with $n$ even, and $Q$ nonsingular $(\bmod p)$, and let $l$ be a nonnegative integer with $l \leq \frac{n}{2}-2$. Suppose that $p \geq 2^{\frac{2(n+3)}{n-2 l-2}}$. Then there exists a primitive solution to (2) with $x_{i}=0,1 \leq i \leq l$, and $\left|x_{i}\right| \leq$ $2^{\frac{4 n+3}{n-l}} p^{\frac{n}{n-l}}+1$, for $l<i \leq n$.

In the case where $l=0$, the theorem gives us a primitive solution of (2) with $\|\mathbf{x}\| \leq 2^{4+\frac{3}{n}} p$, recovering the type of bound obtained in [8] and [6]. To prove these results we shall use finite Fourier series over $\mathbb{Z}_{p^{2}}$, the modular ring in $p^{2}$ elements. The proof here builds upon the work of [6] and [8].

\section{Basic identities and lemmas}

In this section we shall assume that $n$ is even, $p$ is an odd prime, and that $Q(\mathbf{x})$ is a nonsingular quadratic form $(\bmod p)$ with $\Delta_{p}(Q)= \pm 1$. Let $e_{p^{2}}(\alpha)=e^{2 \pi i \alpha / p^{2}}$. Let $V_{p^{2}}=V_{p^{2}}(Q)$ be the set of zeros of $Q$ contained in $\mathbb{Z}_{p^{2}}^{n}$ and let $Q^{*}(\mathbf{y})$ be the quadratic form associated with inverse of the matrix for $Q(\bmod p)$. For $\mathbf{y} \in \mathbb{Z}_{p^{2}}^{n}$ set

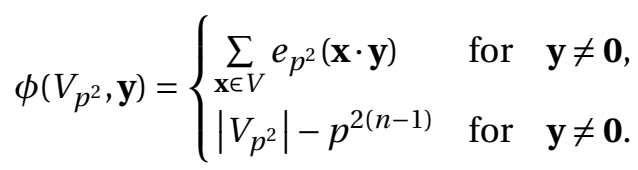

We abbreviate complete sums over $\mathbb{Z}_{p^{2}}^{n}$ and $\mathbb{Z}_{p}^{n}$ in the manner

$$
\sum_{\mathbf{x}}=\sum_{\mathbf{x} \bmod p^{2}}=\sum_{x_{1}=1}^{p^{2}} \cdots \sum_{x_{n}=1}^{p^{2}}, \quad \sum_{\mathbf{x} \bmod p}=\sum_{x_{1}=1}^{p} \cdots \sum_{x_{n}=1}^{p} .
$$

The following lemma gives us a formula for $\phi\left(V_{p^{2}}, \mathbf{y}\right)$. 
Lemma 1. Suppose $n$ is even, $Q$ is nonsingular $(\bmod p)$ and $\Delta=\Delta_{p}(Q)$. For $y \in \mathbb{Z}^{n}$, put $\mathbf{y}^{\prime}=\frac{1}{p} \mathbf{y}$ in case $p \mid \mathbf{y}$ Then for any $\mathbf{y}$,

$$
\phi(V, \mathbf{y})= \begin{cases}p^{n}-p^{n-1} & \text { if } p \nmid y_{i} \text { for some } i \text { and } p^{2} \mid Q^{*}(\mathbf{y}), \\ -p^{n-1} & \text { if } p \nmid y_{i} \text { for some } i \text { and } p \mid Q^{*}(\mathbf{y}), \\ 0 & \text { if } p \nmid y_{i} \text { for some } i \text { and } p \nmid Q^{*}(\mathbf{y}), \\ -\Delta p^{3 n / 2-2}+p^{n-1}(p-1) & \text { if } p \mid y_{i} \text { for all } i \text { and } p \nmid Q^{*}(\mathbf{y}), \\ \Delta(p-1) p^{3 n / 2-2}+p^{n-1}(p-1) & \text { if } p \mid y_{i} \text { for all } i \text { and } p \mid Q^{*}\left(\mathbf{y}^{\prime}\right) .\end{cases}
$$

The proof of Lemma 1 is given (with some work) in Carlitz [14], and in complete detail in [13, Theorem 1].

Let $\alpha(\mathbf{x})$ be a complex valued function defined on $\mathbb{Z}_{p^{2}}^{n}$ with Fourier expansion $\alpha(\mathbf{x})=$ $\sum_{\mathbf{y}} a(\mathbf{y}) e_{p^{2}}(\mathbf{x} \cdot \mathbf{y})$ where $a(\mathbf{y})=p^{-2 n} \sum_{\mathbf{x}} \alpha(\mathbf{x}) e_{p^{2}}(-\mathbf{x} \cdot \mathbf{y})$. Then

$$
\begin{aligned}
\sum_{\mathbf{x} \in V} \alpha(\mathbf{x}) & =\sum_{\mathbf{x} \in V} \sum_{\mathbf{y}} a(\mathbf{y}) e_{p^{2}}(\mathbf{y} \cdot \mathbf{x}) \\
& =\sum_{\mathbf{y}} a(\mathbf{y}) \sum_{\mathbf{x} \in V} e_{p^{2}}(\mathbf{y} \cdot \mathbf{x}) \\
& =a(\mathbf{0})|V|+\sum_{\mathbf{y} \neq \mathbf{0}} a(\mathbf{y}) \sum_{\mathbf{x} \in V} e_{p^{2}}(\mathbf{y} \cdot \mathbf{x}) .
\end{aligned}
$$

Since $a(\mathbf{0})=p^{-2 n} \sum_{\mathbf{x}} \alpha(\mathbf{x})$, we obtain

$$
\left.\sum_{\mathbf{x} \in V} \alpha(\mathbf{x})=p^{-2 n}|V| \sum_{\mathbf{x}} \alpha(\mathbf{x})+\sum_{\mathbf{y} \neq \mathbf{0}} a(\mathbf{y}) \phi\left(V_{p^{3}}, \mathbf{0}\right), \mathbf{y}\right)
$$

Also by noticing that $|V|=\phi\left(V_{p^{2}}, \mathbf{0}\right)+p^{2(n-1)}$, we obtain that

$$
\sum_{\mathbf{x} \in V} \alpha(\mathbf{x})=p^{-2} \sum_{\mathbf{x}} \alpha(\mathbf{x})+\sum_{\mathbf{y}} a(\mathbf{y}) \phi(V, \mathbf{y})
$$

Inserting the value of $\phi\left(V_{p^{2}}, \mathbf{y}\right)$ from Lemma 1 in (4) we obtain (see [8, Lemma 2])

Lemma 2 (The Fundamental Identity). Suppose $n$ is even, $Q$ is nonsingular $(\bmod p)$ and $\Delta=\Delta_{p}(Q)$. Then, for any complex valued $\alpha(\mathbf{x})$ on $\mathbb{Z}_{p^{2}}^{n}$,

$$
\begin{aligned}
\sum_{\mathbf{x} \in V} \alpha(\mathbf{x})= & p^{-2} \sum_{\mathbf{x}} \alpha(\mathbf{x})+p^{n} \sum_{p^{2} \mid Q^{*}(\mathbf{y})} a(\mathbf{y})-p^{n-1} \sum_{p \mid Q^{*}(\mathbf{y})} a(\mathbf{y}) \\
& -\Delta p^{(3 n / 2)-2} \sum_{y_{i}^{\prime}=1}^{p} a\left(p \mathbf{y}^{\prime}\right)+\Delta p^{(3 n / 2)-1} \sum_{p\left|y_{i}, p\right| Q^{*}\left(\mathbf{y}^{\prime}\right)}^{p} a\left(p \mathbf{y}^{\prime}\right) .
\end{aligned}
$$




\section{Proof of main results in the case where $\Delta_{p}(Q)=1$}

Let $\mathscr{B}$ be the box of points in $\mathbb{Z}^{n}$ given by

$$
\mathscr{B}=\left\{\mathbf{x} \in \mathbb{Z}^{n} \mid a_{i} \leq x_{i}<a_{i}+m_{i}, 1 \leq i \leq n\right\},
$$

where $m_{i}=q_{i} p+r_{i}, \quad 0 \leqslant r_{i}<p$ and $q_{i}, r_{i} \in \mathbb{Z}$. Thus the cardinality $\mathscr{B}$ is $|\mathscr{B}|=\prod_{i=1}^{n} m_{i}$. Consider the congruence

$$
Q(\mathbf{x}) \equiv 0 \quad(\bmod p)
$$

Our first step is to obtain an upper bound on the number of solutions of (7) contained in $\mathscr{B}$. First, we treat the case where all $m_{i} \leqslant p$. In this case we can view the box $\mathscr{B}$ in (6) as a subset of $\mathbb{Z}_{p}^{n}$ and appeal to the following result of Cochrane [1, Lemma 3].

Lemma 3. Suppose that $\Delta_{p}(Q)=1$. Let $\mathscr{B}$ be a box of type (6) with all $m_{i} \leqslant p$, and $V_{p}=V_{p}(Q)$ denote the set of zeros of (7) in $\mathbb{Z}_{p}^{n}$. Then

$$
\left|\mathscr{B} \cap V_{p}\right| \leqslant 2^{n}\left(\frac{|\mathscr{B}|}{p}+p^{n / 2}\right) .
$$

Next we consider larger boxes where the $m_{i}$ may exceed $p$. We define

$$
N_{\mathscr{B}}=\prod_{i=1}^{n}\left(\left[\frac{m_{i}}{p}\right]+1\right) .
$$

Partition the box $\mathscr{B}$ in (6) into $N=N_{\mathscr{B}}$ smaller boxes $B_{i}$,

$$
\mathscr{B}=B_{1} \cup B_{2} \cup \cdots \cup B_{N},
$$

where each $B_{i}$ has all of its edge lengths $\leqslant p$. Thus Lemma 3 can be applied to each $B_{i}$. We obtain

$$
\begin{aligned}
\left|\mathscr{B} \cap V_{p, \mathbb{Z}}\right| & =\sum_{i=1}^{N}\left|B_{i} \cap V_{p}\right| \\
& \leqslant \sum_{i=1}^{N} 2^{n}\left(\frac{\left|B_{i}\right|}{p}+p^{n / 2}\right) \\
& =\frac{2^{n}}{p} \sum_{i=1}^{N}\left|B_{i}\right|+N 2^{n} p^{n / 2} \\
& =2^{n}\left(\frac{|\mathscr{B}|}{p}+N p^{n / 2}\right) .
\end{aligned}
$$

Thus we have proved 
Lemma 4. Suppose that $\Delta_{p}(Q)=1$. Let $V_{p, \mathbb{Z}}=V_{p, \mathbb{Z}}(Q)$ be the set of integer solutions of the congruence (7). Then for any box $\mathscr{B}$ of type (6), we have

$$
\left|\mathscr{B} \cap V_{p, \mathbb{Z}}\right| \leqslant 2^{n}\left(\frac{|\mathscr{B}|}{p}+N_{\mathscr{B}} p^{n / 2}\right),
$$

where $N_{\mathscr{B}}$ as defined in (9).

Let $\mathscr{B}$ be a box of points in $\mathbb{Z}^{n}$ as in (3) centered about the origin with edge lengths $m_{i}:=$ $2 M_{i}+1 \leqslant p^{2}, 1 \leq i \leq n$, and view this box as a subset of $\mathbb{Z}_{p^{2}}^{n}$. Let $\chi_{\mathscr{B}}$ be its characteristic function with Fourier expansion $\chi_{\mathscr{B}}(\mathbf{x})=\sum_{\mathbf{y}} a_{\mathscr{B}}(\mathbf{y}) e_{p^{2}}(\mathbf{x} \cdot \mathbf{y})$. Let $\alpha(\mathbf{x})=\chi_{\mathscr{B}} * \chi_{\mathscr{B}}=\sum_{\mathbf{y}} a(\mathbf{y}) e_{p^{2}}(\mathbf{x} \cdot$ y). Then for any $\mathbf{y} \in \mathbb{Z}_{p^{2}}^{n}$,

$$
a(\mathbf{y})=p^{-2 n} \prod_{i=1}^{n} \frac{\sin ^{2} \pi m_{i} y_{i} / p^{2}}{\sin ^{2} \pi y_{i} / p^{2}},
$$

where the term in the product is taken to be $m_{i}$ if $y_{i}=0$. In particular, if we take $\left|y_{i}\right| \leqslant p^{2} / 2$ for all $i$, then using the fact that $|\sin (x)| \geqslant \frac{2}{\pi}|x|$ for $|x| \leqslant \pi / 2$, we have

$$
a(\mathbf{y}) \leqslant p^{-2 n} \prod_{i=1}^{n} \min \left\{m_{i}^{2},\left(\frac{p^{2}}{2 y_{i}}\right)^{2}\right\} .
$$

Since $\mathscr{B}$ is centered about the origin, the Fourier coefficients $a(\mathbf{y})$ are positive real numbers (as can be seen by (11)). Thus by applying the Fundamental Identity (5) to $\alpha(\mathbf{x})=\chi_{\mathscr{B}} * \chi_{\mathscr{B}}$, we obtain

$$
\begin{aligned}
\sum_{\mathbf{x} \in V} \alpha(\mathbf{x}) & \geqslant \underbrace{p^{-2} \sum_{\mathbf{x}} \alpha(\mathbf{x})}_{\text {Main Term }}-\underbrace{p^{n-1} \sum_{p \mid Q^{*}(\mathbf{y})} a(\mathbf{y})}_{E_{1}}-\underbrace{p^{(3 n / 2)-2} \sum_{\mathbf{y}(\bmod p)} a(p \mathbf{y})}_{E_{2}} \\
& \geqslant \text { Main Term }-E_{1}-E_{2} .
\end{aligned}
$$

The main term in (13) is

$$
p^{-2} \sum_{\mathbf{x}} \alpha(\mathbf{x})=p^{-2} \sum_{\mathbf{x}} \chi_{\mathscr{B}} * \chi_{\mathscr{B}}(\mathbf{x})=\frac{|\mathscr{B}|^{2}}{p^{2}},
$$

and the others are error terms. We proceed to bound these error terms.

First, we consider

$$
E_{1}=p^{n-1} \sum_{Q^{*}(\mathbf{y}) \equiv 0(\bmod p)} a(\mathbf{y}) .
$$

Let $\sum^{*}$ be an abbreviation for $\sum_{Q^{*}(\mathbf{y}) \equiv 0(\bmod p),\left|y_{i}\right|<p^{2} / 2}$. Define $\delta_{i}$ by

$$
\delta_{i}= \begin{cases}2^{k_{i}-1} & \text { for } k_{i} \geq 1 \\ 0 & \text { for } k_{i}=0\end{cases}
$$


Using (12) yields

$$
\begin{aligned}
\sum_{\substack{Q^{*}(\mathbf{y}) \equiv 0(\bmod p) \\
\left|y_{i}\right| \leq p^{2} / 2}}|a(\mathbf{y})| & \leq \sum_{k_{1}=0}^{\infty} \cdots \sum_{k_{n}=0}^{\infty} \sum_{\substack{\mathbf{y} \\
\delta_{i} \frac{p^{2}}{m_{i}} \leq\left|y_{i}\right| \leq 2^{k_{i}} \frac{p^{2}}{m_{i}}}}^{*} \prod_{i=1}^{n} \min \left\{\frac{m_{i}^{2}}{p^{2}}, \frac{p^{2}}{4 y_{i}^{2}}\right\} \\
& \leq \sum_{k_{1}=0}^{\infty} \cdots \sum_{k_{n}=0}^{\infty} \sum_{\substack{\mathbf{y} \\
\left|y_{i}\right| \leq 2^{k_{i}} \frac{p^{2}}{m_{i}}}}^{*} \prod_{i=1}^{n} \frac{p^{2}}{4\left(2^{k_{i}-1} p^{2} / m_{i}\right)^{2}} \\
& =\frac{|\mathscr{B}|^{2}}{p^{2 n}} \sum_{k_{1}=0}^{\infty} \cdots \sum_{k_{n}=0}^{\infty} \sum_{\substack{\mathbf{y} \\
\left|y_{i}\right| \leq 2^{k_{i}} \frac{p^{2}}{m_{i}}}}^{*} \prod_{i=1}^{n} \frac{1}{2^{2 k_{i}}} .
\end{aligned}
$$

For non-negative integers $k_{1}, k_{2}, \ldots, k_{n}$, let

$$
\mathscr{B}^{\prime}=\left\{\mathbf{y} \in \mathbb{Z}_{p^{2}}^{n}:\left|y_{i}\right| \leq 2^{k_{i}} \frac{p^{2}}{m_{i}}, 1 \leq i \leq n\right\} .
$$

Put

$$
m_{i}^{\prime}=2\left[\frac{2^{k_{i}} p^{2}}{m_{i}}\right]+1
$$

so that

$$
\left|\mathscr{B}^{\prime}\right|=\prod_{i=1}^{n} m_{i}^{\prime} \leq \prod_{i=1}^{n}\left(\frac{2^{k_{i}+1} p^{2}}{m_{i}}+1\right) \leq \prod_{i=1}^{n} \frac{2^{k_{i}+2} p^{2}}{m_{i}}=4^{n} \frac{p^{2 n}}{|\mathscr{B}|} \prod_{i=1}^{n} 2^{k_{i}} .
$$

Now, from the upper bound (10), we have

$$
\left|\mathscr{B}^{\prime} \cap V_{p, \mathbb{Z}}\right| \leq 2^{n} \frac{\left|\mathscr{B}^{\prime}\right|}{p}+2^{n} N_{\mathscr{B}^{\prime}} p^{n / 2},
$$

where by utilizing (9),

$$
N_{\mathscr{B}^{\prime}}=\prod_{i=1}^{n}\left(\left[\frac{m_{i}^{\prime}}{p}\right]+1\right)=\prod_{\substack{i=1 \\ 2^{k_{i}} \geq \frac{m_{i}}{4 p}}}^{n}\left(\left[\frac{m_{i}^{\prime}}{p}\right]+1\right)
$$

The last equality in (19) follows, since

$$
2^{k_{i}}<\frac{m_{i}}{4 p} \Rightarrow \frac{2^{k_{i}+1} p^{2}}{m_{i}}+1<p \quad \Rightarrow \quad m_{i}^{\prime}<p .
$$

But the right-hand side of (19), is less than or equal to

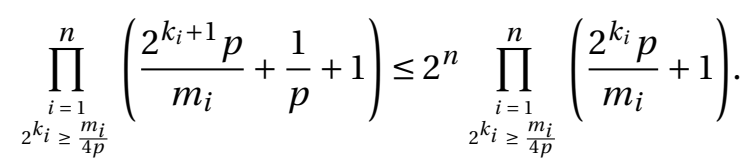


It follows that

$$
N_{\mathscr{B}^{\prime}} \leq 2^{n} \prod_{\substack{i=1 \\ 2^{k} i \geq \frac{m_{i}}{4 p}}}^{n}\left(\frac{2^{k_{i}} p}{m_{i}}+1\right) .
$$

Apply the upper bound (18) to the inner sum $\sum_{\mathbf{y}}^{*}$ in (16). This gives

$$
\begin{aligned}
\sum_{\substack{Q^{*}(\mathbf{y}) \equiv 0(\bmod p) \\
\left|y_{i}\right| \leq p^{2} / 2}} a(\mathbf{y}) & =\frac{|\mathscr{B}|^{2}}{p^{2 n}} \sum_{k_{1}=0}^{\infty} \cdots \sum_{k_{n}=0}^{\infty}\left|\mathscr{B}^{\prime} \cap V_{p, \mathbb{Z}}\right| \prod_{i=1}^{n} \frac{1}{2^{2 k_{i}}} \\
& \leq \frac{|\mathscr{B}|^{2}}{p^{2 n}} \sum_{k_{1}=0}^{\infty} \cdots \sum_{k_{n}=0}^{\infty}\left(2^{n} \frac{\left|\mathscr{B}^{\prime}\right|}{p}+2^{n} N_{\mathscr{B}^{\prime}} p^{n / 2}\right) \prod_{i=1}^{n} \frac{1}{2^{2 k_{i}}} \\
& =\sigma_{1}+\sigma_{2},
\end{aligned}
$$

say. By employing the inequality (17), we find that

$$
\begin{aligned}
\sigma_{1} & =\frac{|\mathscr{B}|^{2}}{p^{2 n}} \sum_{k_{1}=0}^{\infty} \cdots \sum_{k_{n}=0}^{\infty}\left(\prod_{i=1}^{n} \frac{1}{2^{2 k_{i}}}\right) \frac{2^{n}\left|\mathscr{B}^{\prime}\right|}{p} \\
& \leqslant \frac{|\mathscr{B}|^{2}}{p^{2 n}} \sum_{k_{1}=0}^{\infty} \cdots \sum_{k_{n}=0}^{\infty}\left(\prod_{i=1}^{n} \frac{1}{2^{2 k_{i}}}\right)\left(\frac{2^{n}}{p} 4^{n} \frac{p^{2 n}}{|\mathscr{B}|} \prod_{i=1}^{n} 2^{k_{i}}\right) \\
& =8^{n} \frac{|\mathscr{B}|}{p} \sum_{k_{1}=0}^{\infty} \cdots \sum_{k_{n}=0}^{\infty}\left(\prod_{i=1}^{n} \frac{1}{2^{k_{i}}}\right) \\
& \leqslant 8^{n} \cdot 2^{n} \frac{|\mathscr{B}|}{p}=16^{n} \frac{|\mathscr{B}|}{p},
\end{aligned}
$$

and by the inequality (21),

$$
\begin{aligned}
\sigma_{2} & =\frac{|\mathscr{B}|^{2}}{p^{2 n}} \sum_{k_{1}=0}^{\infty} \cdots \sum_{k_{n}=0}^{\infty} 2^{n} N_{\mathscr{B}^{\prime}} p^{n / 2} \prod_{i=1}^{n} \frac{1}{2^{2 k_{i}}} \\
& =2^{n} \frac{|\mathscr{B}|^{2}}{p^{2 n}} p^{n / 2} \sum_{k_{1}=0}^{\infty} \cdots \sum_{k_{n}=0}^{\infty} 2^{n} \prod_{\substack{2_{i=1} \\
2^{k_{i} \geq m_{i} / 4 p}}}^{n}\left(\frac{2^{k_{i}} p}{m_{i}}+1\right) \prod_{i=1}^{n} \frac{1}{2^{2 k_{i}}} \\
& =4^{n} \frac{|\mathscr{B}|^{2}}{p^{2 n}} p^{n / 2} \prod_{i=1}^{n}\left[\sum_{\substack{k_{i}=0 \\
2^{k_{i}<m_{i} / 4 p}}}^{\infty} \frac{1}{2^{2 k_{i}}}+\sum_{\substack{k^{k_{i}} \\
{ }^{k_{i}} \geqslant m_{i} / 4 p}}\left(\frac{2^{k_{i}} p}{m_{i}}+1\right) \frac{1}{2^{2 k_{i}}}\right] \\
& \leq \frac{4^{n}|\mathscr{B}|^{2}}{p^{3 n / 2}} \prod_{i=1}^{n}\left[\sum_{k_{i}=0}^{\infty} \frac{1}{2^{2 k_{i}}}+\sum_{\substack{k_{i} \\
2^{k_{i} \geq m_{i} / 4 p}}}^{2^{k_{i}} m_{i}}\right] \\
& =\frac{4^{n}|\mathscr{B}|^{2}}{p^{3 n / 2}} \prod_{i=1}^{n}\left[\frac{4}{3}+\frac{p}{m_{i}} \sum_{\substack{k_{i}=0 \\
k_{i} \geqslant m_{i} / 4 p}}^{\infty} \frac{1}{2^{k_{i}}}\right]
\end{aligned}
$$




$$
\leq \frac{4^{n}|\mathscr{B}|^{2}}{p^{3 n / 2}} \prod_{i=1}^{n}\left[\frac{4}{3}+\min \left(\frac{2 p}{m_{i}}, \frac{8 p^{2}}{m_{i}^{2}}\right)\right] .
$$

Thus by (14), (21), (22) and (23), we have

$$
\begin{aligned}
E_{1} & \leqslant 16^{n} \frac{|\mathscr{B}|}{p} p^{n-1}+\frac{4^{n}|\mathscr{B}|^{2}}{p^{3 n / 2}} p^{n-1} \prod_{i=1}^{n}\left[\frac{4}{3}+\min \left(\frac{2 p}{m_{i}}, \frac{8 p^{2}}{m_{i}^{2}}\right)\right] \\
& =\underbrace{2^{4 n} p^{n-2}|\mathscr{B}|}_{E_{1,1}}+\underbrace{\frac{4^{n}|\mathscr{B}|^{2}}{p^{n / 2+1}} \prod_{i=1}^{n}\left[\frac{4}{3}+\min \left(\frac{2 p}{m_{i}}, \frac{8 p^{2}}{m_{i}^{2}}\right)\right]}_{E_{1,2}},
\end{aligned}
$$

where $E_{1,1}, E_{1,2}$ denote the terms underlined.

Let us now assume that for some positive integer $l$ we have,

$$
m_{1} \leq \cdots \leq m_{l} \leq p<m_{l+1} \leq \cdots \leq m_{n}
$$

Then for $m_{i} \leq p$,

$$
\frac{4}{3}+\min \left(\frac{2 p}{m_{i}}, 8\left(\frac{p}{m_{i}}\right)^{2}\right) \leq \frac{4}{3}+\frac{2 p}{m_{i}} \leq \frac{4 p}{m_{i}}
$$

and for $m_{i}>p$,

$$
\frac{4}{3}+\min \left(\frac{2 p}{m_{i}}, 8\left(\frac{p}{m_{i}}\right)^{2}\right) \leq \frac{4}{3}+2 \leq \frac{10}{3} .
$$

By (26) and (27), we can write

$$
\prod_{i=1}^{n}\left[\frac{4}{3}+\min \left(\frac{2 p}{m_{i}}, \frac{8 p^{2}}{m_{i}^{2}}\right)\right] \leq \prod_{i=1}^{l} \frac{4 p}{m_{i}} \cdot \prod_{i=l+1}^{n} \frac{10}{3}=\frac{4^{l} p^{l}}{\prod_{i=1}^{l} m_{i}}\left(\frac{10}{3}\right)^{n-l} \leq \frac{4^{n} p^{l}}{\prod_{i=1}^{l} m_{i}},
$$

and consequently (using (23) and (28)),

$$
E_{1,2} \leq \frac{4^{n}|\mathscr{B}|^{2}}{p^{n / 2+1}} \frac{4^{n} p^{l}}{\prod_{i=1}^{l} m_{i}}=\frac{2^{4 n} \prod_{i=1}^{n} m_{i}^{2}}{p^{n / 2-l+1} \cdot \prod_{i=1}^{l} m_{i}}=2^{4 n} p^{l-(n / 2)-1}|\mathscr{B}| \prod_{i=l+1}^{n} m_{i} .
$$

Therefore, by inequalities (24) and (29), we arrive at

$$
E_{1} \leq \underbrace{2^{4 n} p^{n-2}|\mathscr{B}|}_{E_{1,1}}+\underbrace{2^{4 n} p^{l-(n / 2)-1}|\mathscr{B}| \prod_{i=l+1}^{n} m_{i}}_{E_{1,2}} .
$$

We next estimate the error term $E_{2}$, but to do that and also for future reference, we first prove

Lemma 5. Let $\mathscr{B}$ be any box of type (3) and suppose that $\alpha(\mathbf{x})=\chi_{\mathscr{B}} * \chi_{\mathscr{B}}(\mathbf{x})$, and that condition (25) holds. Then we have

$$
\sum_{\mathbf{y} \in \mathbb{Z}_{p}^{n}} a(p \mathbf{y}) \leqslant 2^{n-l} p^{l-2 n}|\mathscr{B}| \prod_{i=l+1}^{n} m_{i}
$$


Proof. We first observe,

$$
\begin{aligned}
\sum_{y_{i}=1}^{p} a(p \mathbf{y}) & =\sum_{y_{i}=1}^{p} \sum_{x_{i}=\mathbf{1}}^{p^{2}} \frac{1}{p^{2 n}} \alpha(\mathbf{x}) e_{p^{2}}(-\mathbf{x} \cdot p \mathbf{y}) \\
& =\sum_{x_{i}=1}^{p^{2}} \frac{1}{p^{2 n}} \alpha(\mathbf{x}) \sum_{y_{i}=1}^{p} e_{p}(-\mathbf{x} \cdot \mathbf{y}) \\
& =\sum_{x_{i}=1}^{p^{2}} \frac{p^{n}}{p^{2 n}} \alpha(\mathbf{x}) \\
& =\frac{1}{p^{n}} \sum_{\mathbf{x} \equiv 0(\bmod p)} \alpha(\mathbf{x}) \\
& =\frac{1}{p^{n}} \sum_{\substack{\mathbf{u} \in \mathscr{B} \\
\mathbf{u}+\mathbf{v} \equiv 0}} \sum_{\mathbf{v} \in \mathscr{B}} 1 \\
& \leq \frac{1}{p^{n}} \prod_{i=1}^{n} m_{i}\left(\left[\frac{m_{i}}{p}\right]+1\right)
\end{aligned}
$$

To obtain the last inequality in (30), we must count the number of solutions of the congruence

$$
\mathbf{u}+\mathbf{v} \equiv \mathbf{0}(\bmod p)
$$

with $\mathbf{u}, \mathbf{v} \in \mathscr{B}$. In fact for each choice of $\mathbf{v}$, there are at most $\prod_{i=1}^{n}\left(\left[m_{i} / p\right]+1\right)$ choices for $\mathbf{u}$. So the total number of solutions is less than or equal to

$$
\prod_{i=1}^{n} m_{i}\left(\left[\frac{m_{i}}{p}\right]+1\right)
$$

Using the hypothesis (25) then continuing from (30), we have

$$
\begin{aligned}
\sum_{y_{i}=1}^{p} a(p \mathbf{y}) & \leq \frac{1}{p^{n}} \prod_{i=1}^{l} m_{i} \prod_{i=l+1}^{n} m_{i}\left(\frac{m_{i}}{p}+1\right) \\
& \leq \frac{|\mathscr{B}|}{p^{n}} \prod_{i=l+1}^{n}\left(\frac{2 m_{i}}{p}\right) \leq \frac{2^{n-l}|\mathscr{B}|}{p^{2 n-l}} \prod_{i=l+1}^{n} m_{i} .
\end{aligned}
$$

The lemma is established.

Now in view of Lemma 5 , it is clear that the error term $E_{2}$ has the estimate

$$
E_{2}=p^{(3 n / 2)-2} \sum_{\mathbf{y}(\bmod p)} a(p \mathbf{y}) \leqslant 2^{n-l} p^{l-(n / 2)-2}|\mathscr{B}| \prod_{i=l+1}^{n} m_{i} .
$$

The following theorem summarizes the final outcome of our investigation for the error terms 
Theorem 2. Suppose that $n \geq 4$, is even and that $\Delta_{p}(Q)=1, V=V_{p^{2}}(Q)$. Then for any box $\mathscr{B}$ centered at the origin, with sides of length $m_{i}=2 M_{i}+1,1 \leq i \leq n$, satisfying (25), we have

$$
\sum_{\mathbf{x} \in V} \alpha(\mathbf{x}) \geq \frac{|B|^{2}}{p^{2}}-\mid \text { Error } \mid
$$

where

$$
\mid \text { Error } \mid \leq \underbrace{2^{4 n} p^{n-2}|\mathscr{B}|}_{E_{1,1}}+\underbrace{2^{4 n} p^{l-(n / 2)-1}|\mathscr{B}| \prod_{i=l+1}^{n} m_{i}}_{E_{1,2}}+\underbrace{2^{n} p^{l-(n / 2)-2}|\mathscr{B}| \prod_{i=l+1}^{n} m_{i}}_{E_{2}} .
$$

In Theorem 2 we have indicated below each term, the error term bounded by the given value.

Next we compare each error term in Theorem 2 to the main term $|B|^{2} / p^{2}$. To make the left-hand side positive, we make each error term less than $1 / 4$ of the main term. For the error term $E_{1,1}$, we need

$$
\frac{1}{4} \frac{|\mathscr{B}|^{2}}{p^{2}} \geq 2^{4 n} p^{n-2}|\mathscr{B}| \Longleftrightarrow|\mathscr{B}| \geq 2^{4 n+2} p^{n},
$$

and for the error term $E_{1,2}$,

$$
\frac{1}{4} \frac{|\mathscr{B}|^{2}}{p^{2}} \geq 2^{4 n} p^{l-(n / 2)-1}|\mathscr{B}| \prod_{i=l+1}^{n} m_{i} \Longleftrightarrow \prod_{i=1}^{l} m_{i} \geq 2^{4 n+2} p^{l-(n / 2)+1} \Longleftrightarrow \prod_{i=1}^{l} \frac{p}{m_{i}} \leq 2^{-4 n-2} p^{(n / 2)-1} .
$$

Finally for the error term $E_{2}$,

$$
\frac{1}{4} \frac{|\mathscr{B}|^{2}}{p^{2}} \geq 2^{n} p^{l-(n / 2)-2}|\mathscr{B}| \prod_{i=l+1}^{n} m_{i} \Longleftrightarrow|\mathscr{B}| \geq 4 \cdot 2^{n} p^{l-(n / 2)} \prod_{i=l+1}^{n} m_{i} \Longleftrightarrow \prod_{i=1}^{l} \frac{p}{m_{i}} \leq 2^{-(n+2)} p^{(n / 2)} .
$$

Putting the pieces together, we deduce

Theorem 3. Suppose that $n \geq 4$ is even, $\Delta_{p}(Q)=1$ and that $V=V_{p^{2}}(Q)$. Let $\mathscr{B}$ be a box centered at the origin satisfying (25). If $|\mathscr{B}| \geqslant 2^{4 n+2} p^{n}$ and $\prod_{i=1}^{l}\left(p / m_{i}\right) \leqslant 2^{-4 n-2} p^{(n / 2)-1}$ (where L.H.S $=$ 1 if $l=0$ ), then

$$
\sum_{\mathbf{x} \in V} \alpha(\mathbf{x}) \geq \frac{|\mathscr{B}|^{2}}{p^{2}}-\frac{3}{4} \frac{|\mathscr{B}|^{2}}{p^{2}}=\frac{1}{4} \frac{|\mathscr{B}|^{2}}{p^{2}}
$$

In particular,

$$
|V \cap(\mathscr{B}+\mathscr{B})| \geq \frac{|\mathscr{B}|}{4 p^{2}} .
$$

Recall that a solution of (3) is called primitive if some coordinate is not divisible by $p$, i.e $p \nmid x_{i}$ for some $i$. We write $p \mid \mathbf{x}$ for imprimitive points.

Corollary 1. Under the hypothesis of Theorem $3, \mathscr{B}+\mathscr{B}$ contains a primitive solution of (2). 
Proof. We need to show that

$$
\sum_{\mathbf{x} \in V} \alpha(\mathbf{x})>\sum_{\substack{\mathbf{x} \in V \\ p \mid \mathbf{x}}} \alpha(\mathbf{x})
$$

First by Lemma 5,

$$
\begin{aligned}
\sum_{\substack{\mathbf{x} \in V \\
p \mid \mathbf{x}}} \alpha(\mathbf{x}) & =\sum_{\substack{p \mid x_{i}, 1 \leq i \leq n}} \alpha(\mathbf{x})=p^{n} \sum_{y=1}^{p} a(p \mathbf{y}) \leq 2^{n-l} p^{l-n}|\mathscr{B}| \prod_{i=l+1}^{n} m_{i} \\
& =\frac{1}{2^{l}} \cdot \frac{2^{n}|\mathscr{B}|}{p^{n-l}} \prod_{i=l+1}^{n} m_{i} \leq \frac{1}{2^{l}} \cdot \frac{|\mathscr{B}|^{2}}{4 p^{2}} .
\end{aligned}
$$

The last inequality is guaranteed by our hypothesis (Theorem 3) that

$$
\prod_{i=1}^{l} \frac{p}{m_{i}} \leq 2^{-4 n-2} p^{(n / 2)-1} .
$$

More precisely, assume (31), then certainly

$$
\prod_{i=1}^{l} \frac{p}{m_{i}} \leq \frac{p^{(n / 2)-1}}{2^{4 n+2}} \Rightarrow \frac{2^{n}}{p^{n-l}} \prod_{i=l+1}^{n} m_{i}<\frac{|\mathscr{B}|}{4 p^{2}} .
$$

So we have now on the one hand,

$$
\sum_{\substack{\mathbf{x} \in V \\ p \mid \mathbf{x}}} \alpha(\mathbf{x})<\frac{|\mathscr{B}|^{2}}{4 p^{2}}
$$

On the other hand, by Theorem 3, we have

$$
\sum_{\mathbf{x} \in V} \alpha(\mathbf{x}) \geq \frac{|\mathscr{B}|^{2}}{4 p^{2}}
$$

We therefore get

$$
\sum_{\substack{\mathbf{x} \in V \\ p \nmid \mathbf{x}}} \alpha(\mathbf{x}) \geqslant \frac{|\mathscr{B}|^{2}}{4 p^{2}}-\sum_{\substack{\mathbf{x} \in V \\ p \mid \mathbf{x}}} \alpha(\mathbf{x})>0 .
$$

The proof of the corollary is complete.

\section{Proof of Main Results in the case where $\Delta_{p}(Q)=-1$}

Suppose now that $n$ is even and that $\Delta_{p}(Q)=-1$. We first need to produce analogues of Lemmas 3 and 4 as follows; see [7, Lemma 2.9] and [12, Theorem 1].

Lemma 6. Let $\mathscr{B}$ be any box of type (6) with all $m_{i} \leqslant p$, and $V_{p}=V_{p}(Q)$ denote to the set of solutions of (7) in $\mathbb{Z}_{p}^{n}$. Then

$$
\left|\mathscr{B} \cap V_{p}\right| \leqslant 2^{n+1}\left(\frac{|\mathscr{B}|}{p}+p^{n / 2}\right)
$$


Lemma 7. Let $V_{p, \mathbb{Z}}=V_{p, \mathbb{Z}}(Q)$ be the set of integer solutions of the congruence (7). Then for any box of type (6),

$$
\left|\mathscr{B} \cap V_{p, \mathbb{Z}}\right| \leqslant 2^{n+1}\left(\frac{|\mathscr{B}|}{p}+N_{\mathscr{B}} p^{n / 2}\right),
$$

where $N_{\mathscr{B}}$ is given in (9).

Applying the Fundamental Identity (5) to $\alpha(\mathbf{x})=\chi_{\mathscr{B}} * \chi_{\mathscr{B}}$ as in the preceding section, but this time with $\Delta=-1$, we have

$$
\sum_{\mathbf{x} \in V} \alpha(\mathbf{x}) \geqslant \underbrace{p^{-2} \sum_{\mathbf{x}} \alpha(\mathbf{x})}_{\text {Main Term }}-\underbrace{p^{n-1} \sum_{p \mid Q^{*}(\mathbf{y})} a(\mathbf{y})}_{E_{1}}-\underbrace{p^{(3 n / 2)-1} \sum_{\begin{array}{c}
p \mid Q^{*}\left(\mathbf{y}^{\prime}\right) \\
\mathbf{y}^{\prime}(\bmod p)
\end{array}} a\left(p \mathbf{y}^{\prime}\right)}_{E_{3}} .
$$

Next we seek bounds on the error terms in (33). For the error term $E_{1}$ we have already seen in the case $\Delta=1$ how this error term is bounded. The same strategy will work in the case $\Delta=-1$, except we shall make use of the upper bound (32) in Lemma 7 instead of the upper bound (10) in Lemma 4 . We find that

$$
\begin{aligned}
\sum_{\substack{Q^{*}(\mathbf{y}) \equiv(\bmod p) \\
\left|y_{i}\right| \leq p^{2} / 2}}|a(\mathbf{y})|= & \frac{|\mathscr{B}|^{2}}{p^{2 n}} \sum_{k_{1}=0}^{\infty} \cdots \sum_{k_{n}=0}^{\infty}\left|\mathscr{B}^{\prime} \cap V_{\mathbb{Z}}\right| \prod_{i=1}^{n} \frac{1}{2^{2 k_{i}}} \\
\leq & \frac{|\mathscr{B}|^{2}}{p^{2 n}} \sum_{k_{1}=0}^{\infty} \cdots \sum_{k_{n}=0}^{\infty}\left(2^{n+1} \frac{\left|\mathscr{B}^{\prime}\right|}{p}+2^{n+1} N_{\mathscr{B}^{\prime}} p^{n / 2}\right) \prod_{i=1}^{n} \frac{1}{2^{2 k_{i}}} \\
& \vdots \\
\leq & 2 \cdot 16^{n} \frac{|\mathscr{B}|}{p}+\frac{|\mathscr{B}|^{2} 4^{n}}{p^{3 n / 2}} \prod_{i=1}^{n}\left[\frac{4}{3}+\min \left(\frac{2 p}{m_{i}}, \frac{8 p^{2}}{m_{i}^{2}}\right)\right] .
\end{aligned}
$$

Thus, it follows that

$$
E_{1} \leqslant \underbrace{2^{4 n+1} p^{n-2}|\mathscr{B}|}_{E_{1,1}}+\underbrace{\frac{2 \cdot 4^{n}|\mathscr{B}|^{2}}{p^{n / 2+1}} \prod_{i=1}^{n}\left[\frac{4}{3}+\min \left(\frac{2 p}{m_{i}}, \frac{8 p^{2}}{m_{i}^{2}}\right)\right]}_{E_{1,2}} .
$$

Assume (as before) that

$$
m_{1} \leqslant \cdots \leqslant m_{l} \leqslant p<m_{l+1} \leqslant \cdots \leqslant m_{n} .
$$

Then for $m_{i} \leqslant p$,

$$
\frac{4}{3}+\min \left(\frac{2 p}{m_{i}}, 8\left(\frac{p}{m_{i}}\right)\right)^{2} \leqslant \frac{4}{3}+\frac{2 p}{m_{i}} \leqslant \frac{4 p}{m_{i}}
$$


and for $m_{i}>p$,

$$
\frac{4}{3}+\min \left(\frac{2 p}{m_{i}}, 8\left(\frac{p}{m_{i}}\right)^{2}\right) \leqslant \frac{4}{3}+2 \leqslant \frac{10}{3} .
$$

By taking account of these two inequalities, we have

$$
\prod_{i=1}^{n}\left[\frac{4}{3}+\min \left(\frac{2 p}{m_{i}}, \frac{8 p^{2}}{m_{i}^{2}}\right)\right] \leqslant \prod_{i=1}^{l} \frac{4 p}{m_{i}} \cdot \prod_{i=l+1}^{n} \frac{10}{3}=\frac{4^{l} p^{l}}{\prod_{i=1}^{l} m_{i}}\left(\frac{10}{3}\right)^{n-l} \leqslant \frac{4^{n} p^{l}}{\prod_{i=1}^{l} m_{i}} .
$$

Using (34) and (35), we infer that

$$
E_{1,2} \leqslant \frac{2 \cdot 4^{n}|\mathscr{B}|^{2}}{p^{n / 2+1}} \frac{4^{n} p^{l}}{\prod_{i=1}^{l} m_{i}}=\frac{2^{4 n+1} \prod_{i=1}^{n} m_{i}^{2}}{p^{n / 2-l+1} \cdot \prod_{i=1}^{l} m_{i}}=2^{4 n+1} p^{l-(n / 2)-1}|\mathscr{B}| \prod_{i=l+1}^{n} m_{i} .
$$

To estimate the error term $E_{3}$, we just need to apply Lemma 5. It is easily seen that

$$
E_{3}=p^{(3 n / 2)-1} \sum_{\mathbf{y}(\bmod p)} a(p \mathbf{y}) \leqslant 2^{n-l} p^{l-(n / 2)-1}|\mathscr{B}| \prod_{i=l+1}^{n} m_{i} .
$$

Thus, we have established,

Theorem 4. Suppose that $n \geqslant 4$ is even, $\Delta_{p}(Q)=-1$ and that $V=V_{p^{2}}(Q)$. Then for any box $\mathscr{B}$ centered at the origin,

$$
\sum_{\mathbf{x} \in V} \alpha(\mathbf{x}) \geqslant \frac{|\mathscr{B}|^{2}}{p^{2}}-\mid \text { Error } \mid
$$

where

$$
\mid \text { Error } \mid \leqslant \underbrace{2^{4 n+1} p^{n-2}|\mathscr{B}|}_{E_{1,1}}+\underbrace{2^{4 n+1} p^{l-(n / 2)-1}|\mathscr{B}| \prod_{i=l+1}^{n} m_{i}}_{E_{1,2}}+\underbrace{2^{n} p^{l-(n / 2)-1}|\mathscr{B}| \prod_{i=l+1}^{n} m_{i}}_{E_{3}} .
$$

As before, in order to obtain a positive sum we seek conditions such that each error term is less than $1 / 4$ of the main term.

$$
\begin{aligned}
& E_{1,1}: \quad \frac{1}{4} \frac{|\mathscr{B}|^{2}}{p^{2}} \geqslant 2^{4 n+1} p^{n-2}|\mathscr{B}| \Longleftrightarrow|\mathscr{B}| \geqslant 2^{4 n+3} p^{n} . \\
& E_{1,2}: \quad \frac{1}{4} \frac{|\mathscr{B}|^{2}}{p^{2}} \geqslant 2^{4 n+1} p^{l-(n / 2)-1}|\mathscr{B}| \prod_{i=l+1}^{n} m_{i} \Longleftrightarrow \prod_{i=1}^{l} \frac{p}{m_{i}} \leqslant 2^{-4 n-3} p^{(n / 2)-1} . \\
& E_{3}: \quad \frac{1}{4} \frac{|\mathscr{B}|^{2}}{p^{2}} \geqslant 2^{n-l} p^{l-(n / 2)-1}|\mathscr{B}| \prod_{i=l+1}^{n} m_{i} \Longleftrightarrow \prod_{i=1}^{l} \frac{p}{m_{i}} \leqslant 2^{l-n-2} p^{(n / 2)-1} .
\end{aligned}
$$

Thus we obtain, 
Theorem 5. Suppose that $n \geqslant 4$ is even, $\Delta_{p}(Q)=-1$ and that $V=V_{p^{2}}(Q)$. If $\mathscr{B}$ is a box satisfying (25), $|\mathscr{B}| \geqslant 2^{4 n+3} p^{n}$ and $\prod_{i=1}^{l}\left(p / m_{i}\right) \leqslant 2^{-4 n-3} p^{(n / 2)-1}$ (with L.H.S $=1$ if $\left.l=0\right)$, then

$$
\sum_{\mathbf{x} \in V} \alpha(\mathbf{x}) \geqslant \frac{|\mathscr{B}|^{2}}{p^{2}}-\frac{3}{4} \frac{|\mathscr{B}|^{2}}{p^{2}}=\frac{1}{4} \frac{|\mathscr{B}|^{2}}{p^{2}} .
$$

In particular,

$$
|V \cap(\mathscr{B}+\mathscr{B})| \geqslant \frac{|\mathscr{B}|}{4 p^{2}} .
$$

As a consequence of Theorem 5, we have the following analogue of Corollary 1 for primitive solutions; the proof is identical to the proof of Corollary 1.

Corollary 2. Under the hypotheses of Theorem $5, \mathscr{B}+\mathscr{B}$ contains a primitive solution of (2).

\section{Proof of Theorem 1}

Let $p$ be an odd prime, $Q$ be a quadratic form over $\mathbb{Z}$ in $n \geq 4$ variables with $n$ even, and $Q$ nonsingular $\left(\bmod p\right.$ ), and let $l$ be a nonnegative integer with $l \leq \frac{n}{2}-2$. Set $m_{i}=2 M_{i}+1$, $1 \leq i \leq n$. For $0 \leq i \leq l$, set $M_{i}=0$, while for $l<i \leq n$, set $M_{i}=\left\lceil 2^{\frac{4 n+3}{n-l}-1} p^{\frac{n}{n-l}}-\frac{1}{2}\right\rceil$. Then for $1 \leq i \leq l$ we have $m_{i}=1$, while for $l<i \leq n$ we have $m_{i}>2^{\frac{4 n+3}{n-l}} p^{\frac{n}{n-l}}$, and so

$$
|\mathscr{B}|=\prod_{i=l+1}^{n} m_{i}>2^{4 n+3} p^{n},
$$

and

$$
\prod_{i=1}^{l} \frac{p}{m_{i}}=p^{l} \leq 2^{-4 n-3} p^{\frac{n}{2}-1},
$$

for $p \geq 2^{\frac{2(n+3)}{n-2 l-2}}$. Thus the hypotheses of both Corollary 1 and Corollary 2 are satisfied, and so there exists a primitive solution of the congruence $Q(\mathbf{x}) \equiv 0\left(\bmod p^{2}\right)$ in the box $\mathscr{B}+\mathscr{B}$, that is a solution with $x_{i}=0,1 \leq i \leq l$ and for $l<i \leq n$,

$$
\left|x_{i}\right| \leq 2 M_{i}=2\left\lceil 2^{\frac{4 n+3}{n-l}-1} p^{\frac{n}{n-l}}-\frac{1}{2}\right\rceil \leq 2^{\frac{4 n+3}{n-l}} p^{\frac{n}{n-l}}+1
$$

\section{Acknowledgements}

The authors would like to sincerely thank the anonymous referee for very valuable and helpful comments and suggestions which made the paper more accurate and readable. 


\section{References}

[1] T. Cochrane, Small zeros of quadratic forms modulo p, J. Number Theory, 33(1989), 286-292.

[2] T. Cochrane, Small zeros of quadratic forms modulo p, II, Proceedings of the Illinois Number Theory Conference, (1989), Birkhäuser, Boston (1990), 91-94.

[3] T. Cochrane, Small zeros of quadratic forms modulo p, III, J. Number Theory, 33 (1991), 92-99.

[4] T. Cochrane, Small zeros of quadratic congruences modulo pq, Mathematika, 37 (1990), 261-272.

[5] T. Cochrane, Small zeros of quadratic congruences modulo pq, II, J. Number Theory 50 (1995), $299-308$.

[6] T. Cochrane and A. Hakami, Small zeros of quadratic congruences modulo $p^{2}$, Proceedings of the American Mathematical Society, 140 (2012), 4041-4052.

[7] A. Hakami, Small zeros of quadratic congruences to a prime power modulus, PhD thesis, Kansas State University, 2009.

[8] A. Hakami, Small zeros of quadratic forms modulo $p^{2}$, JP J. Algebra, Number Theory and Applications, 17 (2011), 141-162.

[9] A. Hakami, Small zeros of quadratic forms modulo $p^{3}$, Advances and Applications in Mathematical Sciences, 9 (2011), 47-69.

[10] A. Hakami, Small primitive zeros of quadratic forms modulo $p^{m}$, The Ramanujan J (2014), DOI 10.1007/s11139-014-9614-3.

[11] A. Hakami, On Cochrane's estimate for small zeros of quadratic forms modulo p, Far East J. Math. Sciences, 50 (2011), 151-157.

[12] A. Hakami, An upper bound for the number of integral solutions of quadratic forms modulo $p$, J. Algebra, Number Theory: Advances and Applications, 6 (2011), 1-17.

[13] A. Hakami, Weighted quadratic partitions $\left(\bmod p^{m}\right)$, A new formula and new demonstration, Tamaking J. Math., 43 (2012), 11-19.

[14] L. Carlitz, Weighted quadratic partitions $\left(\bmod p^{r}\right)$, Math Zeitschr. Bd, 59 (1953), 40-46.

[15] D. R. Heath-Brown, Small solutions of quadratic congruences, Glasgow Math. J, 27 (1985), 87-93.

[16] D.R. Heath-Brown, Small solutions of quadratic congruences II, Mathematika, 38 (1991), $264-284$.

[17] A. Schinzel, H.P. Schlickewei and W.M. Schmidt, Small solutions of quadratic congruences and small fractional parts of quadratic forms, Acta Arithmetica, 37 (1980), 241-248.

[18] Y. Wang, On small zeros of quadratic forms over finite fields, Algebraic structures and number theory (Hong Kong, 1988), 269—-274, World Sci. Publ., Teaneck, NJ, 1990.

[19] Y. Wang, On small zeros of quadratic forms over finite fields, J. Number Theory, 31 (1989), 272-284.

[20] Y. Wang, On small zeros of quadratic forms over finite fields II, A Chinese summary appears in Acta Math. Sinica, 37 (1994), 719-720. Acta Math. Sinica (N.S.), 9 (1993), 382-389.

Department of Mathematics, Jazan University, P.O.Box 277, Jazan, Postal Code: 45142, Saudi Arabia.

E-mail: aalhakami@jazanu.edu.sa 